\title{
The GJR-GARCH and EGARCH option pricing models which incorporate the Piterbarg methodology
}

\author{
Coenraad C.A. Labuschagne ${ }^{1,{ }^{*}, \text { Sven T. von Boetticher }}{ }^{2}$ \\ 1,2Department of Finance and Investment Management, \\ University of Johannesburg, PO Box 524, \\ Aucklandpark 2006, South Africa. \\ *coenraad.labuschagne@gmail.com
}

\begin{abstract}
Knowledge of the risk-neutral distribution of the cumulative return with respect to the model used, is needed in European option pricing. Duan, Gauthier, Simonato and Sasseville (DGSS) provided an analytic approximation model for the case where an EGARCH or a GJR-GARCH specification is used to describe the asset price dynamics. The DGSS model requires the risk-neutral pricing methodology of the Black-Scholes-Merton (BSM) option pricing model. The Global Financial Crisis (GFC) exposed the shortcomings of the risk-neutral option pricing methodology. In this paper, the DGSS option pricing models are extended to incorporate the Piterbarg option pricing methodology.
\end{abstract}

Keywords: Asset pricing, EGARCH, GJR-GARCH, Piterbarg model.

\section{Introduction}

Knowledge of the risk-neutral distribution of the cumulative return with respect to the model used, is needed in European option pricing. GARCH models are known to provide superior performances when calculating asset returns. There is a plethora of GARCH (Bollerslev (1986) [2], Glosten el al. (1993) [4], and Nelson (1991) [6]) models and unfortunately their risk-neutral distributions are not known in all cases. Duan, Gauthier, Simonato and Sasseville (2006) [3] (DGSS) provided an analytic approximation model for the case where an EGARCH or a GJR-GARCH specification is used to describe the asset price dynamics. The DGSS model requires the risk-neutral pricing methodology of the Black-Scholes-Merton (BSM) option pricing model (Black and Scholes (1973) [1] and Merton (1973) [5]).

The Global Financial Crisis (GFC) of 2008 had a significant impact on the financial industry, and on the pricing of derivatives. Before the crisis, the well-known Black-Scholes-Merton (BSM) framework for the pricing of derivatives dictated that the price of a derivative $V_{t}$ is equal to the future expected payoff of the derivative, under the risk-neutral measure, discounted at the risk-free rate, i.e.

$$
V_{t}=e^{-r T} E_{\tilde{Q}}\left[V_{T} \mid \mathscr{F}_{t}\right]
$$

where $V_{T}$ is the payoff of the derivative at maturity $T, r$ is the constant risk-free interest rate, and the expectation is under the risk-neutral measure $\tilde{Q}$, at which the underlying asset price process drifts at the risk-free interest rate, and has a filtration $\mathcal{F}$ set at time $t$. GARCH processes, which intrinsically model the conditional volatility of an asset, were transformed from a real-world measure $P$ into the risk-neutral measure of the BSM framework.

Since the GFC the pricing of derivatives has changed significantly. Due to the default of large banks and corporations such as Lehman Brothers, new frameworks for the pricing of derivatives were constructed, one such framework is given by Piterbarg (2010) [7]. The GFC showed that even large corporations could default, which emphasised the need to include procedures in the pricing of 
derivatives to account for counter-party risk. Piterbarg therefore included the posting of collateral in the pricing of derivatives, which additionally requires that the assumption of a risk-free rate be replaced by multiple interest rates. Hence, the GARCH processes which were adapted to the risk-neutral measure need to be redefined to the new measure of the Piterbarg framework.

In this paper asymmetric GARCH processes under consideration are transformed to be consistent with the Piterbarg methodology, and used to price options in the Piterbarg framework. The Piterbarg methodology entails three unique interest rates to price options. Additionally, the price of options under this methodology depend on collateral payments throughout the lifetime of the option. The risk-neutral measure is replaced by a measure in which the underlying asset drifts at the rate earned on a repurchase agreement, and not the risk-free interest rate. The risk-neutral asset price return dynamics under the risk-neutral measure provided by DGSS do not hold, and a new asset price return dynamic is derived in order to price options using GARCH processes to model the underlying assets returns in the Piterbarg methodology.

\section{The Piterbarg framework}

The Piterbarg framework is a modern extension to the Black-Scholes-Merton framework which relaxes the assumption of a risk-free interest rate and implements three unique deterministic rates, namely a unsecured funding rate $r_{F}$ at which the derivative is funded, the collateral rate $r_{C}$ earned on the posted collateral, and the rate earned on a repurchase agreement in which the hedging position is entered in order to gain the interest rate given by $r_{R}$. The measure used in the Piterbarg framework will be referred to as the $Q_{r_{R}}$ measure, and is the measure under which no arbitrage exists when entering the replicating portfolio into a repurchase agreement. In general we have that $r_{C} \leq r_{R} \leq r_{F}$. The price, $V_{t}$ at time $t$ of a derivative is then given by

$$
V_{t}=E_{Q_{r_{R}}}\left[e^{-\int_{t}^{T} r_{F}(u) d u} V(T)+\int_{t}^{T} e^{-\int_{t}^{u} r_{F}(v) d v}\left(r_{F}(u)-r_{C}(u)\right) \gamma_{C}(u) d u \mid \mathcal{F}_{t}\right]
$$

which, by applying the Feynman-Kac theorem, can be rewritten as

$$
V_{t}=E_{Q_{r_{R}}}\left[e^{-\int_{t}^{T} r_{F}(u) d u} V(T) \mid \mathcal{F}_{t}\right]-E_{Q_{r_{R}}}\left[\int_{t}^{T} e^{-\int_{t}^{u} r_{F}(v) d v}\left(r_{F}(u)-r_{C}(u)\right)\left(V(u)-\gamma_{C}(u)\right) d u \mid \mathcal{F}_{t}\right]
$$

where $\gamma_{C}(s)$ equals the collateral amount paid at time $s \in[t, T]$ and the expectation is taken under the $Q_{r_{R}}$ measure at which the underlying asset grows at the rate $r_{R}$ earned on a repurchase agreement. The price of a derivative in the Piterbarg framework depends on the collateral amount paid at each point in time.

\section{GARCH processes in the Piterbarg measure}

Consider a discrete time economy and let $S_{t}$ be the asset price at time $t$. Under the real world measure $P$ the one period rate of return is assumed to be conditionally lognormally distributed, so that

$$
\ln \left[\frac{S_{t}}{S_{t-1}}\right]=r_{R}+\lambda \sqrt{h_{t}}-\frac{1}{2} h_{t}+\varepsilon_{t}
$$


where $\varepsilon_{t}$ has zero mean and conditional variance $h_{t}$ under measure $P$ and $r_{R}$ is the constant one period repurchase rate of return, and $\lambda$ is the constant unit risk premium. We assume that $\varepsilon_{t}$ follows a $\operatorname{GARCH}(\mathrm{p}, \mathrm{q})$ process under $P$. Using alternative specifications for $h_{t}$ will not change the basic option pricing results as long as conditional normality remains in place. The Piterbarg measure $Q_{r_{R}}$ under which the underlying asset price process drifts at the repurchase rate, needs to satisfy

$$
E_{Q_{r_{R}}}\left[\frac{S_{t}}{S_{t-1}} \mid \mathcal{F}_{t-1}\right]=e^{r_{R}},
$$

and

$$
\operatorname{Var}_{Q_{Q_{R}}}\left[\frac{S_{t}}{S_{t-1}} \mid \mathcal{F}_{t-1}\right]=\operatorname{Var}_{P}\left[\frac{S_{t}}{S_{t-1}} \mid \mathcal{F}_{t-1}\right],
$$

almost surely with respect to measure $P$. This ensures that the one period return of the asset return is equal to the one period return under Piterbarg's $Q_{r_{R}}$ measure, and we require the conditional variances under the two measures to be equal, because one can observe and therefore estimate the conditional variance under $P$.

We denote by $U\left(C_{t}\right)$ and $C_{t}$ the utility function and the aggregate consumption at time $t$, respectively. The parameter $\rho$ denotes the impatience factor. The standard expected utility maximisation argument leads to the following Euler equation:

$$
S_{t-1}=E_{P}\left[e^{-\rho} \frac{U^{\prime}\left(C_{t}\right)}{U^{\prime}\left(C_{t-1}\right)} S_{t} \mid \mathscr{F}_{t-1}\right] .
$$

Let $Y_{t}=v+Z_{t}$ where $v$ is the constant mean and $Z_{t}$ is distributed normally with mean zero and constant variance under $P$. We define the measure $Q_{r_{R}}$ by

$$
d Q_{r_{R}}=e^{\left(r_{R}-\rho\right) T+\sum_{i=1}^{T} Y_{i}} d P
$$

The change of measure allows equation (4) to hold true, while the variance of the returns of the $Q_{r_{R}}$ measure and the $P$ measure are equivalent. This implies that $\varepsilon_{t}=\xi_{t}-\lambda \sqrt{h_{t}}$, where $\xi_{t}$ is a $Q_{r_{R}}$-normal random variable. Upon substitution into the conditional variance equation, it results in the following theorem.

Theorem 3.1 Under the $Q_{r_{R}}$ measure, the log-returns of the underlying asset are given by

$$
\ln \left[\frac{S_{t}}{S_{t-1}}\right]=r_{R}-\frac{1}{2} h_{t}+\xi_{t},
$$

where

$$
\xi_{t} \mid \mathcal{F}_{t-1} \sim N\left(0, h_{t}\right)
$$


and $h_{t}$ is given by any adaptation of a GARCH process, such as the EGARCH or GJR-GARCH processes.

\section{GJR-GARCH and EGARCH in the Piterbarg framework}

Having defined the log-return processes of an asset under the Piterbarg $Q_{r_{R}}$ measure by equation (9), we can write the return dynamics of an asset in the Piterbarg $Q_{r_{R}}$ measure as

$$
\ln \left[\frac{S_{t+1}}{S_{t}}\right]=r_{R}-\frac{1}{2} h_{t+1}+\sqrt{h_{t+1}} \varepsilon_{t},
$$

where $\left\{\varepsilon_{t}: t \in\{1,2, \ldots\}\right\}$ is a sequence of standard normal variables, and the GJR-GARCH and EGARCH processes are chosen to model the conditional volatility of the underlying, which, under the $Q_{r_{R}}$ measure, are given by

$$
h_{t+1}=\beta_{0}+h_{t}\left[\beta_{1}+\beta_{2}\left(\varepsilon_{t}-\lambda\right)^{2}+\beta_{3} \max \left(\lambda-\varepsilon_{t}, 0\right)^{2}\right]
$$

for the GJR-GARCH process, and

$$
\ln \left(h_{t+1}\right)=\beta_{0}+\beta_{1} \ln \left(h_{t}\right)+\beta_{2}\left[\left|\varepsilon_{t}-\lambda\right|+\gamma\left(\varepsilon_{t}-\lambda\right)\right]
$$

for the EGARCH process, respectively.

The GARCH processes under the $Q_{r_{R}}$ measure are used in a Monte-Carlo simulation to find the underlying asset price at the maturity of the option, $T$, and are found recursively by

$$
S_{T}=S_{t} \exp \left\{\sum_{i=t}^{T}\left(r_{R}-\frac{1}{2} h_{i}+\sqrt{h_{i}} \varepsilon_{i}\right)\right\} .
$$

The asset price is then used to find the price of the option in the Piterbarg framework, which is found through application of equations (2) and (3).

\section{Results}

The GJR-GARCH and EGARCH processes are implemented in the Piterbarg framework to price European options on the FTSE/JSE Top 40 index (.TOPI). The option is a one month option, and the different interest rates are assumed to be constant and are given by $r_{C}=0.05, r_{R}=0.1$, and $r_{F}=0.2$. The option prices are found for the scenarios in which zero- and full collateral is continuously posted throughout the lifetime of the option. The time series data of the FTSE/JSE Top 40 index consists of closing prices of all trading dates from the $1^{\text {st }}$ of January 2010 until the $10^{\text {th }}$ of June, 2015. The option prices are converted to the Piterbarg implied volatility for comparison purposes.

Fig. 1 displays the implied volatility for fully collateralised options. The characteristic implied volatility skews found in the market are evident and the different models produce similar results. However, the EGARCH calibrated option prices tend to have a flatter implied volatility skew when compared to the GJR-GARCH calibrated implied volatility skew.

In Fig. 2 the implied volatility of options for which zero collateral is posted are shown. Again, the implied volatility skew of the EGARCH calibrated option prices is flatter than the GJR-GARCH 
calibrated implied volatility skew. The simulations show that the option for which zero collateral is posted is cheaper than the option for which full collateral is posted continuously. This, in turn, is consistent with the Piterbarg methodology.

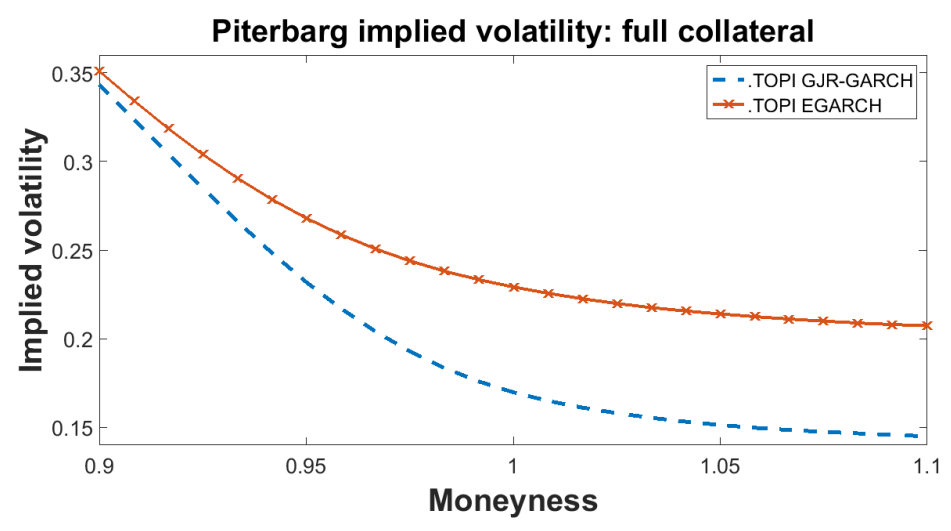

Figure1: The implied volatility of the GJR-GARCH and EGARCH calibrated option prices in the Piterbarg framework, for one month options on the .TOPI, for the case where the trade is fully collateralised continuously.

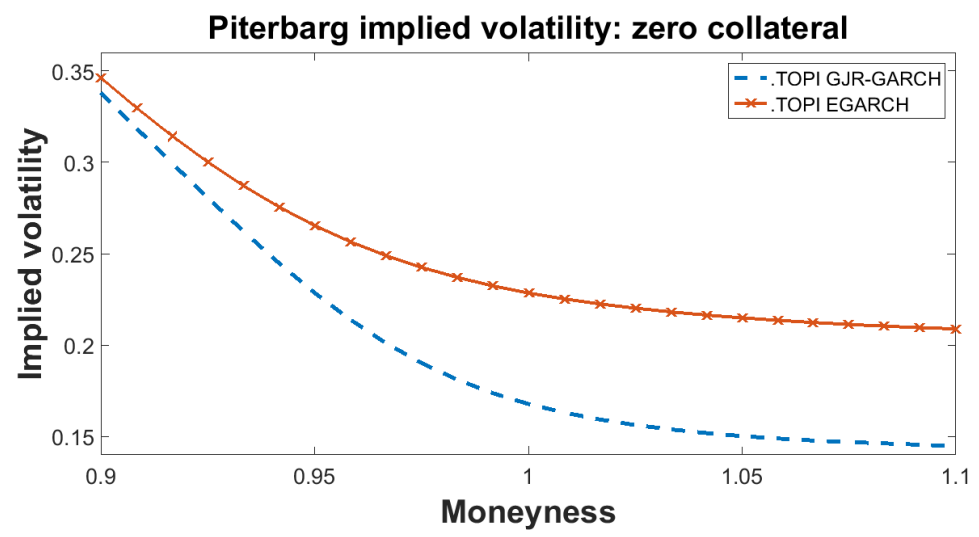

Figure2:The implied volatility of the GJR-GARCH and EGARCH calibrated option prices in the Piterbarg framework, for one month options on the .TOPI, for the case where no collateral is posted throughout the lifetime of the option.

\section{Conclusion}

In this paper the standard GARCH processes were transformed to be consistent with the $Q_{r_{R}}$ measure of the Piterbarg framework. Both the GJR-GARCH and the EGARCH processes where used to simulate the asset returns of the FTSE/JSE Top 40 index, which, through a Monte-Carlo simulation, were used to price one month options on the index. These option prices were converted to the implied volatility of the Piterbarg framework. The EGARCH process generally produces implied volatility skews which are flatter in shape than the GJR-GARCH calibrated volatility skews. Both the EGARCH and the GJR-GARCH models keep the characteristic volatility skew which can be observed in the markets. 


\section{References}

[1] Black, F. and Scholes, M., 1973. The pricing of options and corporate liabilities. The Journal of Political Economy, pp.637-654.

[2] Bollerslev, T., 1986. Generalized autoregressive conditional heteroskedasticity. Journal of Econometrics, 31(3), pp.307-327.

[3] Duan, J., Gauthier, G., Simonato, J. and Sasseville, C., 2006. Approximating the GJR-GARCH and EGARCH option pricing models analytically. Journal of Computational Finance, 9(3), p.41.

[4] Glosten, L.R., Jagannathan, R. and Runkle, D.E., 1993. On the relation between the expected value and the volatility of the nominal excess return on stocks. The Journal of Finance, 48(5), pp.1779-1801.

[5] Merton, R.C., 1973. Theory of rational option pricing. The Bell Journal of Economics and Management Science, pp.141-183.

[6] Nelson, D.B., 1991. Conditional heteroskedasticity in asset returns: A new approach. Econometrica: Journal of the Econometric Society, pp.347-370.

[7] Piterbarg, V., 2010. Funding beyond discounting: collateral agreements and derivatives pricing. Risk, 23(2), p.97. 